\title{
Minimum cost spanning tree games and spillover stability
}

\author{
Ruud Hendrickx · Jacco Thijssen · Peter Borm
}

(C) The Author(s) 2011. This article is published with open access at Springerlink.com

\begin{abstract}
This article discusses interactive minimum cost spanning tree problems and argues that the standard approach of using a transferable utility game to come up with a fair allocation of the total costs has some flaws. A new model of spillover games is presented, in which each player's decision whether or not to cooperate is properly taken into account.
\end{abstract}

Keywords Minimum cost spanning tree problems - Transferable utility games · Spillovers

\section{JEL Classification $\quad \mathrm{C} 71 \cdot \mathrm{C} 72$}

\section{Introduction}

In recent years, much attention has been paid to cooperative aspects of operations research (OR) problems. When multiple players are involved in a particular OR problem, they do not only face the task of optimising some objective function, they also have to allocate the proceeds of cooperation in a fair way (see Borm et al. 2001 for a survey). This article focuses on one such problem, the minimum cost spanning tree (mcst) problem as introduced by Claus and Kleitman (1973). In an mcst problem,

\footnotetext{
R. Hendrickx $(\varangle) \cdot$ P. Borm

Center and Department of Econometrics and Operations Research, Tilburg University, PO Box 90153, 5000 LE Tilburg, The Netherlands

e-mail: ruud@uvt.nl

J. Thijssen

Department of Economics \& Related Studies, University of York, York, UK
} 
players are located in the nodes of a graph with weighted edges and the objective is to connect the players to some source as cheaply as possible.

The predominant paradigm for analysing cooperative OR situations is the model of transferable utility (TU) games. Such a game assigns to each coalition of players a worth, which represents the optimal total value (in an mcst problem, minimal total costs) if the members of that coalition decide to cooperate. This game is then subsequently used to assess whether an allocation rule (i.e. a function assigning to each instance of the problem a division of the total costs) is fair. The bargaining problem to determine an allocation is not explicitly modelled, although some cooperative solution concepts take this bargaining process implicitly into account (e.g. the bargaining set introduced by Aumann and Maschler (1964)). So, any strategic consideration a player might have in such a cooperative situation is only up to a point internalised in the allocation rule.

This article deals with the most basic strategic consideration a player faces: whether or not to cooperate. In an mcst problem, a player might have an incentive not to cooperate, let other players connect themselves to the source and then free-ride using the network that is already in place. In a TU setting, this phenomenon is not taken into account and one simply assumes that all players have to get involved in cooperation. In a TU game, all coalitions get assigned a value that results from cooperation. What happens to the players outside a particular coalition, however, is not taken into account. The model we propose in this paper assigns not only a value to each possible coalition, but also a vector of payoffs to the players who are not in the coalition. This implies that each player can compare his (marginal) contribution to a coalition with the payoff he would get if he stays out of the coalition. In the latter case he might be able to pick the fruits of cooperation by the players in the coalition, without incurring the costs.

The literature offers various cooperative models of the coalition formation process, where each player's decision whether to cooperate is (implicitly) taken into account. One often applies the partition function form (cf. Bloch 1996; Ray and Vohra 1999). The model of partition function games, however, misses an ingredient that is crucial in our context: we do not only have a coalition of cooperating players (possibly consisting of just a single player) and a group of non-cooperating individuals outside, the cooperating coalition also plays a special role in that it builds connections which other players can use.

To properly capture such a structure in which there is one coalition of players cooperating and a group of non-cooperating players outside, we introduce the model of spillover games. The spillover of a coalition to a particular outside player is the payoff (in either reward or cost) to this player if the coalition forms. In our context, the spillover equals the minimum cost of the player connecting himself to the source, given that the coalition has already done so.

We argue that mcst solutions that seem fair in a TU setting (ie, that lie in the core in the corresponding mcst game), might not be particularly appealing when one takes spillovers into account. The aim of this article is not to provide a full model predicting what will happen in the allocation process, but to pinpoint an essential shortcoming of the TU approach in this very primitive strategic setting and to show that our model of spillover games goes some way to remedy these problems. 
The main question that arises is whether it is still possible for the coalition of all players to cooperate and find an allocation of the total costs such that no player has an incentive to leave the coalition and pay his spillover. We provide a characterisation of when such an allocation, which we call spillover stable, exists in terms of the underlying costs on the edges.

The structure of this article is as follows. In Sect. 2, we briefly outline the mcst problem and define the corresponding spillover game. Section 3 discusses a concavity property and show that this property is satisfied by mcst spillover games. In Sect. 4, we discuss spillover stability and characterise the existence of spillover stable allocations in mcst games. Section 5 provides some further applications of the spillover model in other OR games.

\section{Minimum cost spanning tree games and spillovers}

A mcst problem (cf. Claus and Kleitman 1973) is a triple $(N, *, w)$, where $N=$ $\{1, \ldots, n\}$ is a set of players, $*$ is a source and $w: E_{N^{*}} \rightarrow \mathbb{R}_{+}$is a nonnegative weight function, where $N^{*}=N \cup\{*\} . E_{S}$ is defined as the set of all edges between pairs of elements of $S \subset N^{*}$, so that $\left(S, E_{S}\right)$ is the complete graph on $S$ :

$$
E_{S}=\{\{i, j\} \mid i, j \in S, i \neq j\} .
$$

We assume that $w$ satisfies the triangle inequality: $w(\{i, j\}) \leq w(\{i, k\})+w(\{k, j\})$ for all triples $\{i, j, k\} \subset N^{*}$.

With each mcst problem $(N, *, w)$. we associate a corresponding mcst game $(N, c)$, which is a transferable utility cost game, where $c(\emptyset)=0$ and for all $S \subset N, S \neq$ $\emptyset, c(S)$ represents the minimal costs of a tree on $S^{*}=S \cup\{*\}$ :

$$
c(S)=\min \left\{\sum_{e \in R} w(e) \mid R \subset E_{S^{*}} \text { and }\left(S^{*}, R\right) \text { is a tree }\right\} .
$$

The following example illustrates the concept of mcst problem and its corresponding game.

Example 2.1 Consider the mcst problem $(N, *, w)$ given in the following picture:

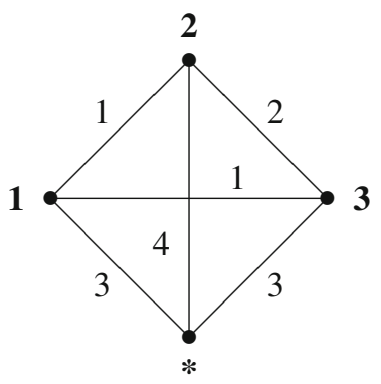


For coalition $N$, there are two optimal trees: $\mathcal{T}_{1}=\{\{*, 1\},\{1,2\},\{1,3\}\}$ and $\mathcal{T}_{2}=$ $\{\{*, 3\},\{3,1\},\{1,2\}\}$, both having costs 5 . The cheapest way for the players of coalition $\{2,3\}$ to connect themselves to the source without passing through player 1 is via the tree $\{\{*, 3\},\{3,2\}\}$, with costs 5 . The mcst game is given in the following table:

\begin{tabular}{c|cccccccc}
$S$ & $\emptyset$ & $\{1\}$ & $\{2\}$ & $\{3\}$ & $\{1,2\}$ & $\{1,3\}$ & $\{2,3\}$ & $N$ \\
\hline$c(S)$ & 0 & 3 & 4 & 3 & 4 & 4 & 5 & 5
\end{tabular}

Bird (1976) proposed a class of solutions for mcst problems: given an mcst, each player pays the costs of his unique adjacent link towards the source. So, the Bird solutions for the problem in Example 2.1 are $(3,1,1)$ (corresponding to $\left.\mathcal{T}_{1}\right)$ and $(1,1,3)$ (corresponding to $\mathcal{T}_{2}$ ). Each Bird solution lies in the core of the corresponding mest game, which is defined by

$$
C(c)=\left\{x \in \mathbb{R}^{N} \mid \sum_{i \in N} x_{i}=c(N), \forall_{S \subset N}: \sum_{i \in S} x_{i} \leq c(S)\right\}
$$

Core elements are stable in the sense that if such an allocation for the grand coalition is proposed, no subcoalition has an incentive to split off, build a tree of their own connecting them to the source and be cheaper off.

One aspect, however, is missing in the mcst game. One can always easily find an allocation that is stable against subgroups of players cooperating, but what about the strategic consideration of players not to cooperate?

Consider the position of player 1 in Example 2.1. If the Bird allocation $(3,1,1)$ is proposed, player 1 can argue that this is unfair to him and not only because there exist core elements that are more favourable to him. Suppose he withdraws from cooperation and lets players 2 and 3 sort it out for themselves. If either of them (or both) connects himself to the source, player 1 can free-ride and make his connection cheaply with costs 1 . So, why would he ever agree to cooperating and paying more than 1 ?

In order to tackle these strategic considerations, we add an extra ingredient to the mcst game: spillovers. In the context of mcst problems, the spillover of a coalition $S \subset N$ to a player $i \notin S$, denoted by $z_{i}^{S}$, is defined as the minimal costs of player $i$ connecting himself to the source, given that coalition $S$ has already done so, so

$$
z_{i}^{S}=\min \left\{w(\{i, j\}) \mid j \in S^{*}\right\}
$$

The mcst game $(N, c)$ and the spillovers $z$ together constitute an mcst spillover (cost) game $(N, c, z)$. In the following example, we illustrate this game for the mcst problem in Example 2.1. 
Example 2.2 Consider the mcst problem $(N, *, w)$ given in Example 2.1. The mcst spillover game is given in the following table.

\begin{tabular}{c|cccccccc}
$S$ & $\emptyset$ & $\{1\}$ & $\{2\}$ & $\{3\}$ & $\{1,2\}$ & $\{1,3\}$ & $\{2,3\}$ & $N$ \\
\hline$c(S)$ & 0 & 3 & 4 & 3 & 4 & 4 & 5 & 5 \\
$z_{1}^{S}$ & 3 & & 1 & 1 & & & 1 & \\
$z_{2}^{S}$ & 4 & 1 & & 2 & & 1 & & \\
$z_{3}^{S}$ & 3 & 1 & 2 & & 1 & & &
\end{tabular}

Note that $z_{i}^{\emptyset}$ simply equals $c(\{i\})$, the cost of connecting player $i$ directly to the source.

We should point out that a spillover game is not a (special case of a) game in partition function form (cf. Myerson 1977). In a game in partition function form, the value of each coalition depends on how the players are partitioned into coalitions. Given the discrete partition $\{\{1\},\{2\},\{3\}\}$, however, we do not know the coalition value of $\{3\}$ in Example 2.2. If coalition $\{3\}$ connects itself, this value is 3 , if coalition $\{1\}$ builds the tree, the spillover of player 3 equals 1 and if coalition $\{2\}$ connects itself to the source, it equals 2 . So, we do not only have a partition into a coalition and remaining singletons, we also assign to one of these coalitions a special role. Since this last ingredient is missing in the model of partition function form games, spillover games cannot be embedded into that class.

\section{Concavity}

A transferable utility game $(N, c)$ is called concave (cf. Shapley 1971) if

$$
c(S \cup U)-c(S) \geq c(T \cup U)-c(T)
$$

for all $U \subset N, S \subset T \subset N \backslash U$. One of the interpretations of concavity is that if a coalition $S$ decides to join another coalition $U$, its extra costs are bigger than when a larger coalition $T$ decides to join $U$.

In our framework with spillovers, the expression $c(S \cup U)-c(S)$ does not represent the additional costs if $S$ decides to join $U$ any more. For, if the players in $S$ choose not to join $U$, they cannot optimise and get their coalitional value $c(S)$, but each member $i \in S$ has to pay his spillover $z_{i}^{U}$. Hence, when spillovers are present, we should adopt a new definition of concavity. A spillover game $(N, c, z)$ is spillover concave if

$$
c(S \cup U)-\sum_{i \in S} z_{i}^{U} \geq c(T \cup U)-\sum_{i \in T} z_{i}^{U}
$$

for all $U \subset N, S \subset T \subset N \backslash U$.

Although not all transferable utility mest games are concave, their spillover counterparts are always spillover concave, as is shown in the following proposition. 
Proposition 3.1 All mcst spillover games are spillover concave.

Proof Let $(N, c, z)$ be an mcst spillover game. Let $U \subset N, S \subset T \subset N \backslash U$. Then

$$
c(T \cup U) \leq c(S \cup U)+\sum_{i \in T \backslash S} z_{i}^{U},
$$

because the right hand side equals the costs of a particular spanning tree for $T \cup U$ (the optimal tree for $S \cup U$ extended with links from $U \cup\{*\}$ to $T \backslash S$ ), whereas the left hand side equals the costs of the cheapest tree for $T \cup U$. By substracting $\sum_{i \in T} z_{i}^{U}$ from both sides, the assertion follows.

In transferable utility games, requiring (3.1) only for $|U|=1$ is sufficient to ensure concavity (cf. Ichiishi 1981). For spillover concavity, such a loosening of the conditions leads to a (strictly) weaker property. However, spillover concavity can be rewritten in terms of a submodularity property.

Proposition 3.2 A spillover game $(N, c, z)$ is spillover concave if and only if

$$
c(A \cup B)+\sum_{i \in A \cap B} z_{i}^{A \backslash B} \leq c(A)+\sum_{i \in B} z_{i}^{A \backslash B}
$$

for all $A, B \subset N$.

Proof For the "only if" part, assume that $(N, c, z)$ is spillover concave. Let $A, B \subset N$. Take $S=A \cap B, T=B$ and $U=A \backslash B$. Then,

$$
\begin{aligned}
c(A \cup B) & =c(T \cup U) \\
& \leq c(S \cup U)-\sum_{i \in S} z_{i}^{U}+\sum_{i \in T} z_{i}^{U} \\
& =c(A)-\sum_{i \in A \cap B} z_{i}^{A \backslash B}+\sum_{i \in B} z_{i}^{A \backslash B} .
\end{aligned}
$$

For the "if" part, assume that (3.2) holds for all $A, B \subset N$. Let $U \subset N, S \subset T \subset$ $N \backslash U$. Take $A=S \cup U$ and $B=T$. Then,

$$
\begin{aligned}
c(T \cup U) & =c(A \cup B) \\
& \leq c(A)+\sum_{i \in B} z_{i}^{A \backslash B}-\sum_{i \in A \cap B} z_{i}^{A \backslash B} \\
& =c(S \cup U)+\sum_{i \in T} z_{i}^{U}-\sum_{i \in S} z_{i}^{U} .
\end{aligned}
$$

In a similar way as for concavity, we should revise the notion of subadditivity, which for transferable utility games is defined as $c(S \cup T) \leq c(S)+c(T)$ for all $S, T \subset$ 
$N, S \cap T=\emptyset$. The idea behind subadditivity is that it is better for a coalition $T$ to join coalition $S$ than to stay separate. A spillover game $(N, c, z)$ is spillover subadditive if

$$
c(S \cup T) \leq c(S)+\sum_{i \in T} z_{i}^{S}
$$

for all $S, T \subset N, S \cap T=\emptyset$. Using Proposition 3.2, it is readily seen that each spillover concave game is spillover subadditive, so all mcst spillover games are spillover subadditive.

\section{Spillover stability}

In this section, we introduce the concept of spillover stability. Whereas a core allocation (where we simply define $C(c, z)=C(c)$ ) is stable against a coalition splitting off, a spillover stable allocation is stable against a player deciding not to cooperate. If player $i$ does not cooperate, he will have costs equal to $z_{i}^{S}$ for some coalition $S$. If no coalition forms, he will have to pay $z_{i}^{\emptyset}=w(\{i, *\})$. For the moment, we exclude this possibility and assume that if $i$ does not cooperate, a non-empty coalition will always form and connect itself to the source. ${ }^{1}$ by

Given a spillover game $(N, c, z)$, the set of spillover stable allocations is defined

$$
S(c, z)=\left\{x \in \mathbb{R}^{N} \mid \sum_{i \in N} x_{i}=c(N), \underline{s}(c, z) \leq x \leq \bar{s}(c, z)\right\},
$$

where for all $i \in N$,

$$
\underline{s}_{i}(c, z)=\min _{S \subset N \backslash\{i\}, S \neq \emptyset} z_{i}^{S} \quad \text { and } \quad \bar{s}_{i}(c, z)=\max _{S \subset N \backslash\{i\}, S \neq \emptyset} z_{i}^{S} .
$$

The spillover stable set is illustrated in the following two examples.

Example 4.1 Consider the mcst spillover game $(N, c, z)$ in Example 2.2. The lower and upper bounds for the spillover stable set are $\underline{s}(c, z)=(1,1,1)$ and $\bar{s}(c, z)=$ $(1,2,2)$, respectively. So,

$$
S(c, z)=\{(1,2,2)\} .
$$

Note that the Bird solutions, $(3,1,1)$ and $(1,1,3)$, are not spillover stable.

Example 4.2 Consider the mest problem $(N, *, w)$ as described in the following picture:

\footnotetext{
1 As explained in the Introduction, our framework of assumptions on cooperation is not intended as a full and realistic description of how players might behave in an mest problem, but as a minimal starting point to point out some deficiencies in the transferable utility approach.
} 


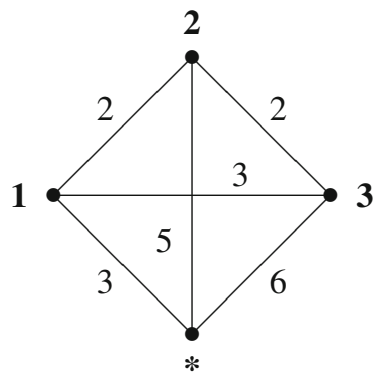

The corresponding spillover game $(N, c, z)$ is given by

\begin{tabular}{c|ccccccc}
$S$ & $\{1\}$ & $\{2\}$ & $\{3\}$ & $\{1,2\}$ & $\{1,3\}$ & $\{2,3\}$ & $N$ \\
\hline$c(S)$ & 3 & 5 & 6 & 5 & 6 & 7 & 7 \\
$z_{1}^{S}$ & & 2 & 3 & & & 2 & \\
$z_{2}^{S}$ & 2 & & 2 & & 2 & & \\
$z_{3}^{S}$ & 3 & 2 & & 2 & & &
\end{tabular}

With $\underline{s}(c, z)=(2,2,2)$ and $\bar{s}(c, z)=(3,2,3)$, we have

$$
S(c, z)=\operatorname{Conv}\{(3,2,2),(2,2,3)\},
$$

where Conv denotes the convex hull. The set of spillover stable allocations is a subset of the core, which equals

$$
C(c, z)=\operatorname{Conv}\{(3,2,2),(3,1,3),(0,1,6),(0,5,2)\} .
$$

The Bird solution, $(3,2,2)$ is spillover stable, while the nucleolus of $(N, c)$ (cf. Schmeidler 1969), $n(c)=\left(1 \frac{1}{3}, 2 \frac{1}{3}, 3 \frac{1}{3}\right)$, is not.

In order to explore the spillover stable set of an mcst game and its relation to the core, we first observe that a player's spillover is monotonic in the coalition with respect to which it is taken.

Lemma 4.1 Let $(N, c, z)$ be an mcst spillover game. For all $i \in N, S \subset T \subset$ $N \backslash\{i\}, z_{i}^{T} \leq z_{i}^{S}$.

As a result of Lemma 4.1, in an mcst spillover game, we have $\underline{s}_{i}(c, z)=z_{i}^{N \backslash\{i\}}$ and $\bar{s}_{i}(c, z)=\max _{j \in N \backslash\{i\}} z_{i}^{\{j\}}$ for all $i \in N$. Note that the lower bound for $i$ equals the costs of the cheapest edge adjacent to $i$, whereas his upper bound equals the minimum of the costs of his most expensive edge to another player and his direct connection to the source.

The following proposition characterises non-emptiness of the spillover stable set.

Proposition 4.2 Let $(N, c, z)$ be an mcst spillover game. Then $S(c, z) \neq \varnothing$ if and only if $\sum_{i \in N} \max _{j \in N \backslash\{i\}} z_{i}^{\{j\}} \geq c(N)$. 
Proof The "only if" part is trivial. For the "if" part, assume that $\sum_{i \in N} \max _{j \in N \backslash\{i\}} z_{i}^{\{j\}}$ $\geq c(N)$. Obviously, $\underline{s}(c, z) \leq \bar{s}(c, z)$. Also, $\sum_{i \in N} z_{i}^{N \backslash\{i\}}=\sum_{i \in N} \min _{j \in N^{*} \backslash\{i\}}$ $w(\{i, j\}) \leq c(N)$. Hence, there exists an $x \in \mathbb{R}^{N}$ with $\sum_{i \in N} x_{i}=c(N)$ such that $\underline{s}_{i}(c, z)=z_{i}^{N \backslash\{i\}} \leq x_{i} \leq \max _{j \in N \backslash\{i\}} z_{i}^{\{j\}}=\bar{s}_{i}(c, z)$ for all $i \in N$.

We observed in Example 4.2 that even if each player's most expensive edge is towards the source, the resulting spillover game can still be spillover stable. However, Proposition 4.2 states that the edges between the players must be sufficiently expensive compared to the edges to the source for a spillover stable allocation to exist.

Although core stability and spillover stability are based on different ideas, for threeplayer games spillover stability is a stronger property than core stability. This is shown in the following proposition.

Proposition 4.3 Let $(N, *, w)$ be an mcst problem with $|N|=3$ and let $(N, c, z)$ be the corresponding spillover game. Then $S(c, z) \subset C(c, z)$.

Proof Let $x \in S(c, z)$. Then $\sum_{i \in N} x_{i}=c(N)$. For all $i \in N, x_{i} \leq \max _{j \in N \backslash\{i\}} z_{i}^{\{j\}} \leq$ $w(\{i, *\})=c(\{i\})$. Also, $x_{i} \geq z_{i}^{N \backslash\{i\}}$ for all $i \in N$, so $\sum_{j \in N \backslash\{i\}} x_{i}=\sum_{j \in N} x_{j}-x_{i} \leq$ $c(N)-z_{i}^{N \backslash\{i\}} \leq c(N \backslash\{i\})$, where the final inequality follows from the observation that $c(N \backslash\{i\})+z_{i}^{N \backslash\{i\}}$ equals the costs of a particular spanning tree for the grand coalition. Hence, $\sum_{i \in S} x_{i} \leq c(S)$ for all $S \subset N$ and $x \in C(c, z)$.

In general, however, the spillover stable set is not a subset of the core and also the reverse inclusion does not hold, as is shown in the following example.

Example 4.3 Consider the mcst problem $(N, *, w)$ as described the following picture:

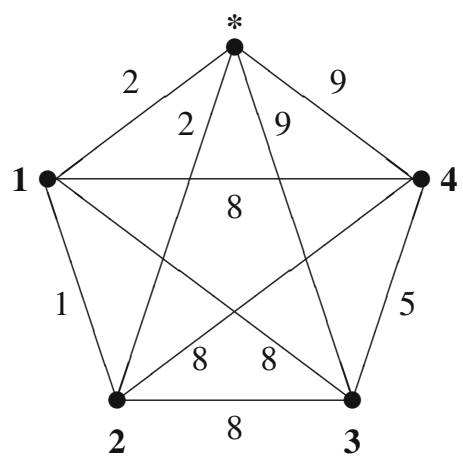

The upper and lower bounds for the spillover stable set are given by $\underline{s}(c, z)=$ $(1,1,5,5)$ and $\bar{s}(c, z)=(2,2,8,8)$. The spillover stable allocation $(2,2,6,6)$ is not an element of the core, because coalition $\{1,2\}$ pays too much. On the other hand, the core element $(1,1,5,9)$ is not spillover stable because of player 4 . The allocation $(1,1,7,7)$ is an element of the spillover core. 


\section{Further research}

Minimum cost spanning tree problems are not the only class of OR problems in which spillovers occur. A related phenomenon arises in travelling salesman problems (Tamir 1989). In a travelling salesman problem, there is a graph in which the vertices represent the locations of the players (and the salesman) and the edges represent the roads between them along which the salesman can travel, where each edge has a nonnegative cost associated with it. The problem is to find a cheapest Hamiltonian circuit in this graph.

Also, each subcoalition faces the same problem of finding a cheapest Hamiltonian circuit through the vertices in which the players in this coalition and the salesman are located. This gives rise to a cooperative cost game. As is the case in mcst problems, however, one does not take into account that there are spillovers involved. If a subcoalition of players decides to work together and invite the salesman to travel to them according to their cheapest tour, the salesman might come near some players outside the coalition, making it cheaper for them to have him come to visit them as well.

In sequencing problems (cf. Curiel et al. 1989), spillovers can also play a role. In a sequencing problem, there is a queue of players waiting to be served. The players in the queue might have different opportunity costs, so moving high-cost players to the front while compensating the low-cost players through side payments can result in a Pareto improvement.

Normally, in such situations, only pairs of players who are adjacent in the queue are allowed to switch, so that a third player can never suffer. If we use our spillover model, however, this restriction is unnecessary, since the effect of any pairwise switch on the other players can be taken into account explicitly.

Acknowledgement Ruud Hendrickx acknowledges financial support from the Netherlands Organisation for Scientific Research (NWO).

Open Access This article is distributed under the terms of the Creative Commons Attribution Noncommercial License which permits any noncommercial use, distribution, and reproduction in any medium, provided the original author(s) and source are credited.

\section{References}

Aumann, R., \& Maschler, M. (1964). The bargaining set for cooperative games. In M. Dresher, L. Shapley, \& A. Tucker (Eds.), Advances in game theory (pp. 443-476). Princeton: Princeton University Press.

Bird, C. (1976). On cost allocation for a spanning tree: A game theoretic approach. Networks, 6, 335-350.

Bloch, F. (1996). Sequential formation of coalitions in games with externalities and fixed payoff division. Games and Economic Behavior, 14, 90-123.

Borm, P., Hamers, H., \& Hendrickx, R. (2001). Operations research games: A survey. TOP, 9, 139-216.

Claus, A., \& Kleitman, D. (1973). Cost allocation for a spanning tree. Networks, 3, 289-304.

Curiel, I., Pederzoli, G., \& Tijs, S. (1989). Sequencing games. European Journal of Operational Research, 40, 344-351.

Ichiishi, T. (1981). Supermodularity: Applications to convex games and the greedy algorithm for LP. Journal of Economic Theory, 25, 283-286.

Myerson, R. (1977). Values for games in partition function form. International Journal of Game Theory, 6, 23-31. 
Ray, D., \& Vohra, R. (1999). A theory of endogenous coalition structures. Games and Economic Behavior, 26, 286-336.

Schmeidler, D. (1969). The nucleolus of a characteristic function game. SIAM Journal of Applied Mathematics, 17, 1163-1170.

Shapley, L. (1971). Cores of convex games. International Journal of Game Theory, 1, 11-26.

Tamir, A. (1989). On the core of a traveling salesman cost allocation game. Operations Research Letters, 8, 31-34. 